\title{
Factor structures of a Japanese version of the Geriatric Depression Scale and its correlation with the quality of life and functional ability.
}

\section{AUTHOR(S):}

Imai, Hissei; Yamanaka, Gaku; Ishimoto, Yasuko; Kimura, Yumi; Fukutomi, Eriko; Chen, Wen-Ling; Matsuoka, Sachi; ... Okumiya, Kiyohito; Otsuka, Kuniaki; Matsubayashi, Kozo

\section{CITATION:}

Imai, Hissei ... [et al]. Factor structures of a Japanese version of the Geriatric Depression Scale and its correlation with the quality of life and functional ability.. Psychiatry research 2014, 215(2): 460-465

\section{ISSUE DATE:}

2014-02-28

URL:

http://hdl.handle.net/2433/182937

\section{RIGHT:}

(c) 2013 Elsevier Ireland Ltd.; This is not the published version. Please cite only the published version.; この論文は出版社版でありません。引 用の際には出版社版をご確認ご利用ください。 


\section{Factors of depressive mood and their relation}

\section{to the quality of life and functional ability in community-dwelling elderly people in Japan}

Hissei Imai* a, Gaku Yamanakab, Yasuko Ishimotoc, Yumi Kimurac, Eriko Fukutomi $^{d}$, Wen-ling Chen ${ }^{d}$, Sachi Matsuoka ${ }^{e}$, Mire Tanaka ${ }^{d}$, Ryota Sakamoto ${ }^{\mathrm{c}, \mathrm{f}}$, Taizo Wada $^{\mathrm{c}}$, Kiyohito Okumiya ${ }^{\mathrm{e}}$, Kuniaki Otsuka ${ }^{\mathrm{b}}$, and Kozo Matsubayashic

a Field Medicine, Graduate School of Medicine, Kyoto University, Kyoto, Japan.

${ }^{\mathrm{b}}$ Medical Center East, Tokyo Women's Medical University, Tokyo, Japan.

${ }^{c}$ The Center for Southeast Asian Studies, Kyoto University, Kyoto, Japan.

'Field Medicine, Graduate School of Public Health, Kyoto University, Kyoto, Japan.

e Graduate School of Asian and African Aria Studies, Kyoto University, Kyoto, Japan.

${ }^{f}$ Hakubi Center, Kyoto University, Kyoto, Japan.

${ }^{g}$ Institute for Humanity and Nature, Kyoto, Japan.

${ }^{*}$ Corresponding author: Hissei Imai, Field Medicine, Graduate School of Medicine, Kyoto University, 46 Shimoadachi-sho, Yoshida, Sakyo-ku, Kyoto 606-8501, Japan (E-mail: ihits@hotmail.com, telephone: +81-75-753-7302, fax: +81-75-753-7350). 


\begin{abstract}
We investigated the 15-item Geriatric Depression Scale (GDS-15) in terms of its factors; reproducibility; and relation to activities of daily living, social factors, medical conditions, and quality of life among community-dwelling elderly people in Japan. The study population consisted of 736 community-dwelling elderly participants aged 65 years or older. Exploratory factor analysis of the data and correlation coefficients between the factors and activities of daily living, quality of life, social factors, and medical condition were calculated in consecutive two years. The reproducibility of the results was also evaluated. As the result, GDS-15 had 3 reproducible factors specified as follows: factor I, "energy loss and pessimistic outlook"; factor II, "positive mental status (reversed)"; and factor III, "empty feeling." Factor I showed greater correlation with quality of life, factor II correlated more with activities of daily living, and factor III correlated more with subjective cognitive function. These results suggest the GDS-15 can be used as a tool for assessing the functional ability and quality of life as well as depressive mood.
\end{abstract}

Keywords: depression, Geriatric Depression Scale, factor analysis, activities of daily living, qol, cognitive function 


\section{Introduction}

Depressive mood is a multifaceted phenomenon involving loss of hope, satisfaction, energy, and interest. It is often accompanied by feelings of boredom, worthlessness, helplessness, and a loss of interest in activities. Many questionnaires have been developed to identify pathological depressive mood. Most of these questionnaires identify depressive mood using a sum of the reported symptoms. However, individual symptoms have been shown to have different qualities. Studies analyzing the Center for Epidemiologic Studies Depression Scale indicate that the loss of a positive affect does not always indicate equal negative affects ${ }^{1,2}$. Studies on the 15-itemGeriatric Depression Scale (GDS-15) reveal that positive psychological affects, specifically the satisfaction with life, happiness, and energy may have a favorable effect on the maintenance of functional ability, regardless of the negative affects $^{3}$. The diagnostic criteria of major depression also considered the different quality of symptoms such as those observed in the melancholic feature seen in patients suffering from major depression. ${ }^{4}$ Accordingly, the factors attributing to depressive mood in questionnaires are as important as the simple summation of symptoms.

The GDS-15 is a self-administered depression questionnaire for elderly people, which omits the non-specific physical symptoms that frequently occur among elderly populations. ${ }^{5}$ The factor structure of the GDS-15 and its relation to functional disability has been investigated in previous studies; however, these studies have critical limitations.

Firstly, most of the studies ${ }^{6 \cdot 9}$ did not confirm the reproducibility of the factor structure, with the number of reported factors differing from 2 to 9 . Meta-analysis demonstrated that 4 factor structures were observed in all samples; however, the 
number of factors ranged from 4 to 6 , and the items composing the factors were different based on the language of the GDS-15. ${ }^{10}$ This implies that the factors presented in these studies lack reproducibility. Further, some of the aforementioned studies performed confirmatory analyses, which can assess the goodness-of-fit, but not the reproducibility of these factors.

Second, to our knowledge, although there are 2 studies on the relationship between the factors of the GDS-15 and functional disabilities, including the basic activities of daily living (ADL) and instrumental activities of daily living (IADL) ${ }^{11,12}$, there are no studies on the relationship between the quality of life (QOL) and the factors of the GDS-15, which should represent the true outcome regarding the depression scale.

The aim of this study is to investigate the reproducibility of the factor structure of the GDS-15 and to show its relation to health-related variables including QOL and physical and social variables.

\section{Methods}

\subsection{Participants}

The study was conducted in 2005-2006, and the study population consisted of 736 community-dwelling elderly participants aged 65 years or older $(\mathrm{M}: \mathrm{F}=$ 406:330, mean age $[S D]=74.3$ [6.6]) living in Tosa (town), Japan. Health questionnaires were distributed by the local government and returned by mail.

T town is situated in the central region of Shikoku, which is the smallest and least populous of Japan's 4 main islands. Its primary industries are agriculture and forestry. During the study period, the town had a population of 4,851 , with 1,822 (37.6\%) residents aged 65 years or older. 
Participants included in the study were those who answered the GDS-15 without omissions in the consecutive years; participants with omissions in one or both years $(53 \%)$ were excluded from the study. Figure 1 illustrates the participant selection procedure.

\subsection{Instrument}

\subsubsection{Geriatric Depression Scale-15}

The GDS-15 is a validated depression scale comprising 15 items. It was developed to exclude the effects of non-specific somatic symptoms such as anorexia and insomnia, which are frequently observed among elderly populations..$^{5,13}$ Each item can have 2 answers, i.e., yes or no; the highest possible score is 15, which indicates the most severe depressive state. Using a cut-point of 5 or more, the GDS-15 has a sensitivity of $97 \%$ and a specificity of $95 \%$, compared with the results of structured clinical interviews for the determination of depression. ${ }^{14}$

\subsubsection{Activities of daily living}

Each subject rated his or her independence with respect to 7 items corresponding to basic activities of daily living (BADL). Specifically, these items were as follows: walking, ascending and descending stairs, feeding, dressing, going to the toilet, bathing, and grooming. Each BADL item was evaluated based on 4 levels of competence: 3, completely independent; 2, requiring some assistance; 1 , requiring much assistance; 0 , completely dependent. The scores for the 7 BADL were summed to a total score $(0-21) .{ }^{15}$

For advanced ADLs, the Tokyo Metropolitan Institute of Gerontology Index of Competence rating scale $(0-13)$ was used to measure competence. ${ }^{16,17}$ This scale 
quantifies instrumental self-maintenance (0-5), intellectual activity (0-4), and social roles (0-4). This assessment consists of a 13-item index including 3 sublevels of competence $^{16,17}$ : (i) instrumental ADL (5 items: the ability to use public transport, buy daily necessities, prepare a meal, pay bills, and handle banking matters; each rated as "yes" or "no"); (ii) intellectual activities (4 items: the ability to complete forms, read newspapers, read books or magazines, and show interest in television programs or news articles on health-related matters; each rated as "yes" or "no"); and (iii) social roles (4 items: the ability to visit friends, give advice to relatives and friends in confidence, visit someone at the hospital, and initiate conversation with younger people; each rated as "yes" or "no").

\subsubsection{Cognitive function}

Cognitive function was subjectively assessed and ranked using an 8-level scale developed for the Nursing Care Insurance Program by the Japanese Ministry of Health, Labor and Welfare. The scale is ranked as follows: 0 , having no cognitive impairment; 1, having slight cognitive impairment but being independent without help in daily life; 2, having mild cognitive impairment but being independent with someone's attention, which is seen mainly outside the house; 3 , having mild cognitive impairment but being independent with someone's attention, which is seen also in the house; 4 , having cognitive impairment and requiring care, which is seen mainly during the day; 5, having cognitive impairment and requiring care, which is seen mainly at night; 6, having cognitive impairment and requiring continuous care; 7 , having cognitive impairment with severe behavior disorder requiring medical care. ${ }^{18}$ Participants ranked as 0 and 1 were considered to be independent with respect to subjective cognitive function. 


\subsubsection{Socio-demographic information}

Socio-demographic variables including age, sex, years of education, loss of partner, and living alone, which are known to be risk factors for the development of major depression, were assessed..$^{19,20}$

\subsection{Analysis}

Statistical analysis was performed using IBM SPSS ver. 20.0 (IBM Inc., Armonk, NY). Exploratory factor analysis using the maximum likelihood analysis with varimax rotation was conducted on the 15 items of the GDS-15. Items with a factor loading of 0.3 or greater were considered to contribute to the factor. If there were items with multiple loadings of 0.3 or greater, the factor with the greatest loading score was considered to be contributed by the items. Correlation coefficients were calculated using Pearson's method and Spearman's method for parametric and nonparametric variables, respectively. P-values less than 0.05 were considered significant.

\section{Results}

Table 1 shows the basic socio-demographic characteristics of the participants. In 2005, the mean age (SD) of the participants was 74.3 (6.6) years, and the percentage of male participants in Tosa (town) was $55.2 \%(n=736)$. The mean BADL score (SD) was 20.1 (2.8), indicating that the participant group was almost entirely independent.

Table 2 shows the results of factor analysis in Tosa (town) during 2005 and 2006. A maximum likelihood analysis was conducted on the 15 items with varimax 
rotation for the respective data in 2 samples. The Kaiser-Meyer-Olkin measure verified the sampling adequacy for the analysis $(\mathrm{KMO}=0.91$ and 0.92 for $\mathrm{T}$ town 2005 and T town 2006, respectively), and all KMO values for individual items were greater than 0.5 . Bartlett's test of sphericity $\left(\chi^{2}(105)=3303.81, \chi^{2}(105)=4178.14\right.$ for $T$ town 2005 and T town 2006, respectively, $p<0.001$ ), revealed that correlations between items were sufficiently large for maximum likelihood analysis. Three factors had eigenvalues larger than Kaiser's criterion of 1 in each data, and in combination, they explained $40.4 \%$ and $40.4 \%$ of the variance ( $T$ town 2005 and $T$ town 2006, respectively). Table 3 summarizes the items reproduced in consecutive years. Factor I comprised 7 items: "prefers to stay at home"; "feels full of energy (reversed)"; "is afraid that something bad will happen"; "believes that most people are better off"; "has dropped activities or interests"; "often feels helpless"; "has problems with memory." Factor II comprised 4 items: "satisfied with life (reversed)"; "in good spirits (reversed)"; "happy most of the time (reversed)"; and "feels wonderful to be alive now (reversed)." Factor III comprised 3 items: "life feels empty," "often gets bored," and "feels present self is worthless." The item "situation is hopeless" was loaded differently in the consecutive years.

Table 4 shows the correlation between factors and variables for consecutive years. The correlation between factors and socio-demographic variables, and between past and present illness were weak. Almost all the factors correlated significantly and moderately with the QOL; however, the correlations between factor II and relationships with family and friends were weak.

\section{Discussion}

There have been 4 studies on factors of the GDS-15 in Japan. 11, 12, 21, 22 
However, their results were inconsistent, with the reported factors ranging from 2 to 4 . Some of these studies evaluated the association between the factors and functional disability, but none of them evaluated the reproducibility of the factors. Other studies on the factor structure of the GDS-15 conducted outside Japan did not investigate its reproducibility, although some studies did perform confirmatory factor analysis, which only showed goodness-of-fit. To the best of our knowledge, this is the first study showing the reproducibility of the factor structure of GDS-15 and its relation to the QOL.

The present study, conducted over 2 consecutive years revealed that the GDS-15 in Japan had 3 replicable factors. Factor I could be defined as "energy loss and pessimistic outlook," factor II as "positive mental status (reversed)," and factor III as "empty feeling."

The factor "energy loss and pessimistic outlook" correlated with the QOL more than other factors. Further, this factor was moderately correlated with all items of QOL, but the relation with subjective happiness was relatively higher than that with other QOL. Items composing the factor "energy loss and pessimistic outlook" should be considered when the focus of evaluation is on the QOL. Especially, as the items "prefers to stay at home," "has dropped activities or interests," and "has problems with memory" can be objectively observed, these questions may be useful in clinical practice or community screening.

The factor "positive mental status" also correlated with the QOL, but it seems to reflect ADLs more than the QOL. In fact, a study showed that the unimpaired positive items of the GDS-15 had a favorable effect on the maintenance of functional ability expressed as BADL. ${ }^{3}$ Another study ${ }^{11}$ also indicated that positive mental status correlated with BADL yielding a correlation coefficient of -0.37 . Our results support 
these findings, but they showed that advanced ADL such as IADL, intellectual activities, and social roles seem to be related more to this factor than to BADL. Further research is required on the predictive value of "positive mental health" against advanced ADLs. The status of ADLs may be inferred by referring items composing this factor.

Most of the correlations between the "empty feeling" factor and the variables investigated in this study were weaker than the correlation with other factors. Only the correlation with independence in subjective cognitive function was stronger for this factor than for the other factors. Interestingly, the factor containing the item "problems with memory" had a weak correlation with independence in subjective cognitive function. One possible explanation is that substantial cognitive impairment may be expressed indirectly in Japan. It is said that depression tends to be denied or expressed somatically in China. ${ }^{23}$ This result may be applied to the expression of cognitive function in Japan. As impaired subjective cognitive function could be expressed as an "empty feeling," we should be mindful of memory function if complaints about an "empty feeling" are observed and vice versa.

This study is limited in that objective functional variables were not included. However, previous research involving patients with parkinsonism revealed that the patients accurately self-reported their disability, even in the presence of depression and cognitive impairment. ${ }^{24}$ Considering this, our results are not misdirected. Another limitation is that investigated variables showed moderate correlation with factors at the most. The present study does not fully explain the meaning of each factor; further study with more variables may be required.

In conclusion, our study revealed that GDS-15 consisted of 3 reproducible factors. The correlation between factors and variables were different; 1 factor showed 
more correlation with the QOL, another factor correlated more with the ADLs, and the last factor correlated more with the subjective cognitive function. These results suggest that the GDS-15 can be used as a tool for assessing functional ability and QOL as well as depressive mood.

\section{Acknowledgement}

We wish to thank all the participants and community workers of $\mathrm{T}$ town in Japan who aided in this study. This study was supported by Grant-in-Aid of Ministry of Health, Welfare and Labor: Choju-Kagaku Kenkyu (No: 005, Leader: Kenji Toba), Grant-in-Aid of Research Institute for Humanity and Nature (3-4 FR). Authors declare no conflict of interest.

\section{References}

1. Iwata N, Umesue M, Egashira $\mathrm{K}$ et al. Can positive affect items be used to assess depressive disorders in the Japanese population? Psychol Med 1998; 28: 153-8.

2. Jang Y, Kwag KH, Chiriboga DA. Not saying I am happy does not mean I am not: cultural influences on responses to positive affect items in the CES-D. J Gerontol B Psychol Sci Soc Sci 2010; 65: 684-90.

3. Hirosaki M, Ishimoto Y, Kasahara Y et al. Positive affect as a predictor of lower risk of functional decline in community-dwelling elderly in Japan. Geriatr Gerontol Int in press.

4. American Psychiatric Association. Diagnostic and statistical manual of mental disorders 
(4th ed., text rev.). Washington, DC, 2000.

5. Yesavage JA, Brink TL, Rose TL et al. Development and validation of a geriatric depression screening scale: a preliminary report. J Psychiatr Res 1982; 17: 37-49.

6. Chiu HF, Lee HC, Wing YK, Kwong PK, Leung CM, Chung DW. Reliability, validity and structure of the Chinese Geriatric Depression Scale in a Hong Kong context: a preliminary report. Singapore Med J 1994; 35: 477-80.

7. Incalzi RA, Cesari M, Pedone C, Carbonin PU. Construct validity of the 15-item geriatric depression scale in older medical inpatients. J Geriatr Psychiatry Neurol 2003; 16: 23-8.

8. Brown PJ, Woods CM, Storandt M. Model stability of the 15-item Geriatric Depression Scale across cognitive impairment and severe depression. Psychol Aging 2007; 22: 372-9.

9. Salamero M, Marcos T. Factor study of the Geriatric Depression Scale. Acta Psychiatr Scand 1992; 86: 283-6.

10. Kim G, Decoster J, Huang CH, Bryant AN. A meta-analysis of the factor structure of the Geriatric Depression Scale (GDS): the effects of language. Int Psychogeriatr 2012: 1-11.

11. Onishi J, Suzuki Y, Umegaki H, Endo H, Kawamura T, Iguchi A. A comparison of depressive mood of older adults in a community, nursing homes, and a geriatric hospital: factor analysis of Geriatric Depression Scale. J Geriatr Psychiatry Neurol 2006; 19: 26-31.

12. Onishi J, Umegaki H, Suzuki Y, Uemura K, Kuzuya M, Iguchi A. The relationship between functional disability and depressive mood in Japanese older adult inpatients. $J$ Geriatr 
Psychiatry Neurol 2004; 17: 93-8.

13. J.I. Sheikh JAY. Geriatric Depression Scale (GDS); recent evidence and development of a shorter version. Haworth Press, New York 1986.

14. Nyunt MS, Fones C, Niti M, Ng TP. Criterion-based validity and reliability of the Geriatric Depression Screening Scale (GDS-15) in a large validation sample of community-living Asian older adults. Aging Ment Health 2009; 13: 376-82.

15. Matsubayashi K, Okumiya K, Wada T, Osaki Y, Doi Y, Ozawa T. Secular improvement in self-care independence of old people living in community in Kahoku, Japan. Lancet 1996; 347: 60 .

16. Ishizaki T, Watanabe S, Suzuki T, Shibata H, Haga H. Predictors for functional decline among nondisabled older Japanese living in a community during a 3-year follow-up. $J$ Am Geriatr Soc 2000; 48: 1424-9.

17. Koyano W, Shibata H, Nakazato K, Haga H, Suyama Y. Measurement of competence: reliability and validity of the TMIG Index of Competence. Arch Gerontol Geriatr 1991; 13: 103-16.

18. Japanese Ministry of Health LaW. A text book for primary nursing care requirement authorization. 2009 .

19. Lehtinen V, Joukamaa M. Epidemiology of depression: prevalence, risk factors and treatment situation. Acta Psychiatr Scand Suppl 1994; 377: 7-10. 
20. Cole MG, Dendukuri N. Risk factors for depression among elderly community subjects: a systematic review and meta-analysis. Am J Psychiatry 2003; 160: 1147-56.

21. Yatomi N. The factor structure and item characteristics of the GDS (Geriatric Depression Scale) short version in a Japanese elderly sample. Gerontol Sociol 1994; 16: 29-36.

22. Schreiner AS, Morimoto T, Asano H. Depressive symptoms among poststroke patients in Japan: frequency distribution and factor structure of the GDS. Int $J$ Geriatr Psychiatry 2001; 16: 941-9.

23. Parker G, Gladstone G, Chee KT. Depression in the planet's largest ethnic group: the Chinese. Am J Psychiatry 2001; 158: 857-64.

24. Brown RG, MacCarthy B, Jahanshahi M, Marsden CD. Accuracy of self-reported disability in patients with parkinsonism. Arch Neurol 1989; 46: 955-9. 
Figure 1. Participant selection and determination during 2005-2006

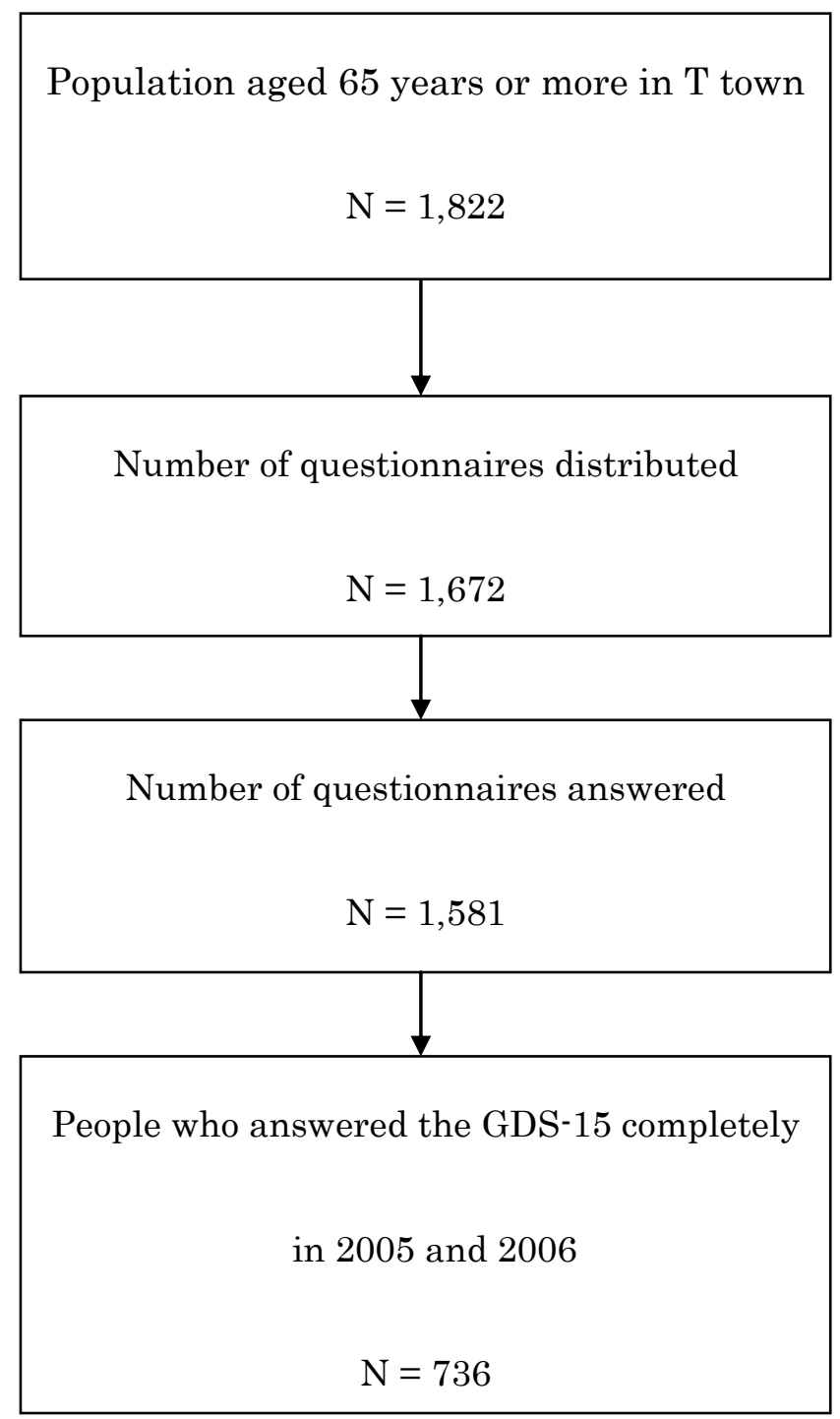


Table1. Basic demography, mental state, quality of life, activities of daily living, and medical conditions.

T town $2005(n=736)$

\begin{tabular}{lc}
\hline Age, mean (SD) & $74.3(6.6)$ \\
Male, \% & 55.2 \\
Loss of partner, \% & 28.4 \\
Living alone, \% & 12.8 \\
Depressive mood and cognitive function & \\
GDS-15 score, mean (SD) & $4.7(3.9)$ \\
Independence in subjective memory function, \% & 96.0 \\
VAS QOLs (0-100), mean (SD) & \\
Family relationship & $79.1(20.2)$ \\
Friend relationship & $75.7(21.0)$ \\
Financial satisfaction & $55.4(24.4)$ \\
Subjective health & $59.4(22.2)$ \\
Subjective happiness & $66.2(22.1)$ \\
ADLs & $3.1(1.1)$ \\
Basic ADL (0-21), mean (SD) & $3.2(1.2)$ \\
Instrumental ADL (0-5), mean (SD) & \\
Intellectual activity (0-4), mean (SD) & \\
Social role (0-4), mean (SD) & \\
Stroke & \\
\hline Diabetes mellitus & \\
\hline
\end{tabular}


Cardiovascular disease

Osteoarthritis

24.9

GDS-15: 15-item Geriatric Depression Scale

VAS: Visual Analogue Scale

ADL: Activities of Daily Living 
Table 2. Factor analysis of 15-item Geriatric Depression Scale in T town between 2005 and 2006

\begin{tabular}{|c|c|c|c|c|c|c|c|}
\hline \multirow[b]{2}{*}{ Item } & & \multicolumn{3}{|c|}{2005} & \multicolumn{3}{|c|}{2006} \\
\hline & & 1 & II & III & 1 & II & III \\
\hline 1 & Satisfied with life (reversed) & .31 & .66 & .22 & .30 & .69 & .19 \\
\hline 2 & Dropped activities, interest & .53 & .17 & .18 & .63 & .18 & .19 \\
\hline 3 & Life is empty & .36 & .28 & .52 & .35 & .33 & .48 \\
\hline 4 & Often bored & .28 & .17 & .55 & .23 & .13 & .58 \\
\hline 5 & In good spirits (reversed) & .05 & .35 & .25 & .04 & .36 & .30 \\
\hline 6 & Afraid something bad will happen & .53 & .24 & .21 & .49 & .25 & .24 \\
\hline 7 & Happy most of the time (reversed) & .21 & .39 & .14 & .26 & .72 & .11 \\
\hline 8 & Often feel helpless & .63 & .12 & .16 & .64 & .18 & .24 \\
\hline 9 & Prefer to stay at home & .39 & -.01 & .20 & .39 & .07 & .24 \\
\hline 10 & Problems with memory & .43 & .09 & .16 & .32 & .08 & .27 \\
\hline 11 & Wonderful to be alive now (reversed) & .19 & .53 & .53 & .21 & .55 & .31 \\
\hline 12 & Feel worthless & .30 & .25 & .65 & .25 & .34 & .58 \\
\hline 13 & Feel full of energy (reversed) & .47 & .27 & .07 & .47 & .25 & .03 \\
\hline 14 & Situation is hopeless & .51 & .28 & .39 & .41 & .33 & .45 \\
\hline 15 & Most people are better off than you & .42 & .27 & .15 & .39 & .34 & .24 \\
\hline Eige & values & 2.4 & 1.9 & 1.7 & 2.3 & 2.1 & 1.7 \\
\hline Prop & rtion of variance explained & 16.3 & 12.5 & 11.6 & 15.2 & 14.0 & 11.2 \\
\hline
\end{tabular}

Method of factor extraction: maximum likelihood solution

Rotation method: varimax rotation

Values in italics and boldface indicate the items composing the factor. 
Table 3. Summary of the reproduced factors of the 15-item Geriatric Depression

Scale in Tosa for 2 consecutive years

\begin{tabular}{ll} 
Factor & \multicolumn{1}{c}{ Tosa } \\
\hline & Prefer to stay at home \\
& Feel full of energy (reversed) \\
& Afraid something bad will happen \\
I $\quad$ Most people are better off than you \\
Dropped activities, interest \\
Often feel helpless \\
Problems with memory \\
Satisfied with life (reversed) \\
In good spirits (reversed) \\
Happy most of the time (reversed) \\
Wonderful to be alive now (reversed) \\
\hline III
\end{tabular}

$\overline{\text { Factor names correspond to those in Table } 2 .}$ 
Table 4. Correlation between factors of GDS-15, socio-demographic variables, ADLs, medical history and QOLs in Tosa (town) for consecutive years

\begin{tabular}{lll}
\hline & 2005 & \\
\hline I II III
\end{tabular}

\begin{tabular}{lll}
\hline & 2006 & \\
\hline I & II & III
\end{tabular}

\begin{tabular}{|c|c|c|c|c|c|c|}
\hline Age & .052 & $.22^{* *}$ & $.26^{* *}$ & .045 & $.19^{* *}$ & $.22^{* *}$ \\
\hline Loss of partner & .036 & -.011 & -.031 & .067 & $.10^{* *}$ & $.078^{*}$ \\
\hline Living alone & $.093^{* *}$ & $.091^{*}$ & $.090^{\star *}$ & $.094^{* *}$ & $.14^{\star \star \star}$ & $.15^{\star \star \star}$ \\
\hline Basic ADL & $-.25^{\star \star \star}$ & $-.33^{* \star *}$ & $-.33^{* \star *}$ & $-.25^{\star \star \star}$ & $-.35^{\star \star \star}$ & $-.37^{* * *}$ \\
\hline Instrumental ADL & $-.27^{* \star *}$ & $-.34^{* * *}$ & $-.28^{* \star *}$ & $-.27^{\star \star \star}$ & $-.35^{\star \star \star}$ & $-.19^{* * \star}$ \\
\hline Intellectual activities & $-.30^{\star \star *}$ & $-.33^{* * *}$ & $-.24^{* \star *}$ & $-.34^{* \star *}$ & $-.37^{\star \star \star}$ & $-.25^{\star \star \star}$ \\
\hline Social role & $-.34^{* * *}$ & $-.36^{* \star *}$ & $-.31^{* \star *}$ & $-.30^{* * *}$ & $-.35^{* \star *}$ & $-.24^{* * *}$ \\
\hline Independence in cognitive function & $-.24^{* * *}$ & $-.29^{\star \star \star}$ & $-.32^{* \star *}$ & $-.24^{\star \star \star}$ & $-.27^{* \star *}$ & $-.37^{* * *}$ \\
\hline Hypertension & .049 & $.079^{*}$ & .052 & .040 & $.071^{*}$ & $.077^{*}$ \\
\hline Stroke & $.16^{\star *}$ & $.12^{* *}$ & $.15^{\star *}$ & $.15^{\star * *}$ & $.20^{* * *}$ & $.16^{* * *}$ \\
\hline Osteoarthritis & $.14^{* * *}$ & $.12^{* *}$ & $17^{\star \star \star}$ & $.12^{* * *}$ & $.13^{\star * *}$ & $.14^{\star * *}$ \\
\hline Subjective health & $-.40^{* * *}$ & $-.39^{* \star *}$ & $-.47^{* \star *}$ & $-.42^{* \star \star}$ & $-.37^{* * *}$ & $-.52^{* * *}$ \\
\hline
\end{tabular}


Family relationship

Friend relationship

Financial satisfaction

Subjective happiness

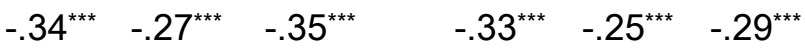

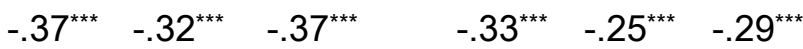

$-.43^{* * *}-.28^{* \star *}-.39^{* *} \quad-.39^{* \star *}-.33^{* \star *}-.36^{* \star *}$

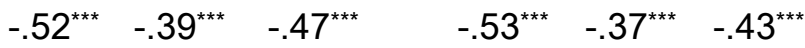

$\overline{\text { Pearson's r and Spearman's r were calculated for correlations of categorical and }}$ continuous variables, respectively. Factor names correspond to Table 2. * $p<$ $0.05,{ }^{* *} p<0.005,{ }^{* * *} p<0.001$ 